Journal of Lake Sciences, Vol. 10, Suppl., pp493-498, 1998.

Copyright (C)1998 by Science Press, Beijing.

Printed in P. R. China. All rights reserved.

\title{
Numerical Prediction of Water Quality Change in Pinqing Lake*
}

\author{
HUANG Ping ${ }^{1}$ and ZHAO Jiguo ${ }^{2}$ \\ 1.Zhongshan University, Guangzhou, 510275,China \\ 2.Guangdong Province Environmental Monitoring Center
}

\begin{abstract}
Pinqing Lake, which is near Shanwei city, Guangdong Province, is a lagoon. Water flow in the lake is controlled by tide current. As area of Shanwei city expands quickly, more and more urban wastewater pours into the lake. The water quality near an outfall is bad consequently. In this paper, numerical models of 2-D horizontal time-varying flow and diffusion equations are developed. Difference methods are exployed to solve the equations. After increasing domestic and industrial loads are consided, the water quality in Pinqing Lake is predicted by using the mathematics models.
\end{abstract}

Keywords: lagoon, mathematics model, difference method, prediction of water quality

\section{Introduction}

Pinqing Lake, which is in the east of Shanwei City, Guangdong Province (Fig.1), is a lagoon. Its area is about $22 \mathrm{~km}^{2}$. Most depth of water in the lake is about 1.5 metres. Water flow in the lake is controlled by tide current.

In recent years, with the economical development the urban area expands rapidly and the urban population increases quickly. More and more industry waste water and domestic sewage discharged into Pinqing Lake. Therefore, the water quality of Pinqing Lake was heavily threatened. For the prediction of influence to water quality of Pinqing Lake by the development of urban

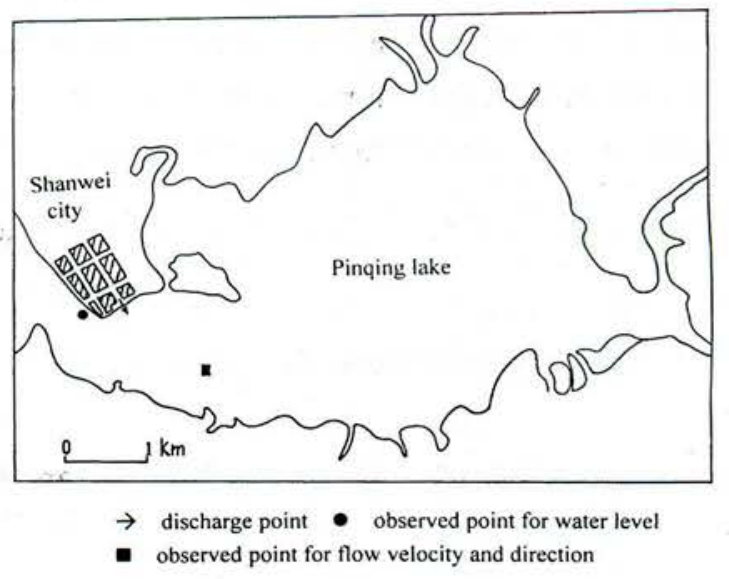

Fig.1 Location map of Pinqing Lake area of Shanwei city, a 2-D time-varying flow equation was developed to simulate the flow of Pinqing Lake. Then, the diffusion equation was used to predict the water quality variety of

- Received 1997-02-25; accepted 1998-03-27. 
Pinqing Lake in the future. These results were useful for the sewage treatment and the water quality protect of Pinqing Lake.

\section{Flow and diffusion equations}

With the hypothesis that the vertical pressure tally with static pressure distribution, compare with gravity the vertical acceleration can be ignored. Assume water density is a constant and wind-force is igored. The flow of Pinqing Lake can be described as the equations $(1) \sim(3)$, The pollutant diffusion can be described as the equation (4).

$$
\begin{aligned}
& \frac{\partial z}{\partial t}+\frac{\partial[u(z+h)]}{\partial x}+\frac{\partial[v(z+h)]}{\partial y}=0 \\
& \frac{\partial u}{\partial t}+u \frac{\partial u}{\partial x}+v \frac{\partial u}{\partial y}=f v-g \frac{\partial z}{\partial x}-g \frac{u \sqrt{u^{2}+v^{2}}}{c^{2}(z+h)}+\xi_{x} \nabla^{2} u \\
& \frac{\partial v}{\partial t}+u \frac{\partial v}{\partial x}+v \frac{\partial v}{\partial y}=-f u-g \frac{\partial z}{\partial y}-g \frac{v \sqrt{u^{2}+v^{2}}}{c^{2}(z+h)}+\xi_{y} \nabla^{2} v \\
& \frac{\partial(H C)}{\partial t}+\frac{\partial(u H C)}{\partial x}+\frac{\partial(v H C)}{\partial y}=\frac{\partial}{\partial x}\left(E_{x} H \frac{\partial C}{\partial x}\right)+\frac{\partial}{\partial y}\left(E_{y} H \frac{\partial C}{\partial y}\right)-k H C+H S
\end{aligned}
$$

where $u$ is the vertically-averaged velocity in the $\mathrm{x}$-direction; $v$ is the vertically-averaged velocity in the $y$-direction; $z$ is the height from the coordinate plane to the water surface (water level); $h$ is the depth from the coordinate plane to the bottom; $f$ is Coriolis parameter; $c$ is the Chezy coefficient ; $g$ is the gravitational acceleration; $t$ is the time variable; $\xi_{x}$ and $\xi_{y}$ are the eddy viscosity coefficients in $x$-direction and in y-direction; $\nabla^{2}=\frac{\partial^{2}}{\partial x^{2}}+\frac{\partial^{2}}{\partial y^{2}} ; H=z+h ; C$ is the concentration of any property; $E_{x}$ and $E_{y}$ are dispersion coefficients; $k$ is the decay coefficient; $S$ is source term.

\section{Finite-difference approximation}

Utilizing a block-centered finite-difference grid,(1) (3) may be approximated by the following forms: 


$$
\begin{aligned}
& z_{i j}^{n+1}=z_{i j}^{n}-\frac{\Delta t}{\Delta x}\left[u_{i+\frac{1}{2}, j}^{n}(\bar{z}+\bar{h})_{i+\frac{1}{2}, j}^{n}-u_{i-\frac{1}{2}, j}^{n}(\bar{z}+\bar{h})_{i-\frac{1}{2}, j}^{n}\right]-\frac{\Delta t}{\Delta y}\left[v_{i, j+\frac{1}{2}}^{n}(\bar{z}+\bar{h})_{i, j+\frac{1}{2}}^{n}\right. \\
& \left.-v_{i, j \frac{1}{2}}^{n}(\bar{z}+\bar{h})_{i, j-\frac{1}{2}}^{n}\right] \\
& {\left[1+\Delta t\left(\frac{\partial u}{\partial x}\right)_{i-\frac{1}{2}, j}^{n}\right] u_{i-\frac{1}{2}, j}^{n+1}=u_{i-\frac{1}{2}, j}^{n}-\bar{v}^{n} \Delta t\left(\frac{\partial u}{\partial y}\right)_{i-\frac{1}{2}, j}^{n}+f \Delta t \bar{v}^{n}-g \frac{\Delta t}{\Delta x}\left(z_{i j}^{n+1}-z_{i-1, j}^{n+1}\right)} \\
& -g \Delta t \frac{u_{i-\frac{1}{2}, j}^{n}\left[\left(u_{i-\frac{1}{2}, j}^{n}\right)^{2}+\left(\bar{v}^{n}\right)^{2}\right]^{\frac{1}{2}}}{\left.\sum_{i-\frac{1}{2}, j}^{n+1}\right)^{2}(\bar{z}+\bar{h})_{i-\frac{1}{2}, j}^{n+1}}+\xi_{x} \Delta t\left(\nabla^{2} u\right)_{i-\frac{1}{2}, j}^{n} \\
& {\left[1+\Delta t\left(\frac{\partial v}{\partial y}\right)_{i-\frac{1}{2}, j}^{n}\right] v_{i, j-\frac{1}{2}}^{n+1}=v_{i, j-\frac{1}{2}}^{n}-\bar{u}^{n} \Delta t\left(\frac{\partial v}{\partial x}\right)_{i, j-\frac{1}{2}}^{n}-f \Delta \bar{u}^{n}-g \frac{\Delta t}{\Delta y}\left(z_{i j}^{n+1}-z_{i-1, j}^{n+1}\right)}
\end{aligned}
$$

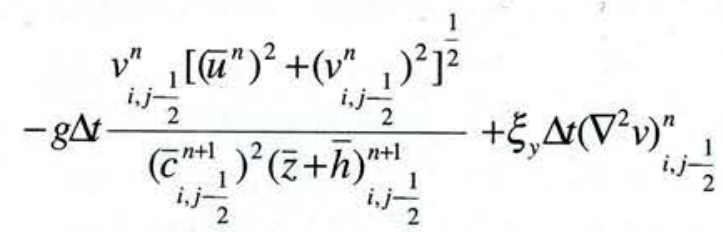

where:

$$
\begin{aligned}
& \bar{u}^{n}=\frac{1}{4}\left(u_{i-\frac{1}{2}, j-1}^{n}+u_{i-\frac{1}{2}, j}^{n}+u_{i+\frac{1}{2}, j-1}^{n}+u_{i+\frac{1}{2}, j}^{n}\right) ; \bar{c}_{i-\frac{1}{2}, j}^{n+1}=\frac{1}{2}\left(c_{i j}^{n+1}+c_{i-1, j}^{n+1}\right) ; \\
& (\bar{z}+\bar{h})_{i-\frac{1}{2}, j}^{n+1}=\frac{1}{2}\left[(z+h)_{i j}^{n+1}+(z+h)_{i-1, j}^{n+1}\right] ;\left(\frac{\partial u}{\partial x}\right)_{i-\frac{1}{2}, j}^{n}=\left(u_{i+\frac{1}{2}, j}^{n}-u_{i-\frac{3}{2}, j}^{n}\right) /(2 \Delta x) ; \\
& \left(\frac{\partial v}{\partial x}\right)_{i, j-\frac{1}{2}}^{n}=\left(v_{i+1, j-\frac{1}{2}}^{n}-v_{i-1, j-\frac{1}{2}}^{n}\right) /(2 \Delta x) ; \\
& \left(\nabla^{2} u\right)_{i-\frac{1}{2}, j}^{n}=\left(u_{i+\frac{1}{2}, j}^{n}+u_{i-\frac{3}{2}, j}^{n}-2 u_{i-\frac{1}{2}, j}^{n}\right) / \Delta x^{2}+\left(u_{i-\frac{1}{2}, j+1}^{n}+u_{i-\frac{1}{2}, j-1}^{n}-2 u_{i-\frac{1}{2}, j}^{n}\right) / \Delta y^{2}
\end{aligned}
$$

Utilizing a characteristic difference form, Eq. (4) may be approximated by the following forms:

$C_{i, j}^{n+1}=\frac{1}{H_{i, j}^{n+1}}\left\{H_{i, j}^{n} C_{i, j}^{n}-\frac{\Delta t}{\Delta x} \Delta(u H C)^{n}-\frac{\Delta t}{\Delta y} \Delta(v H C)^{n}+\frac{\Delta t}{\Delta x}\left(E_{x} H \frac{\Delta C}{\Delta x}\right)^{n}\right.$ 


$$
\left.+\frac{\Delta t}{\Delta y}\left(E_{y} H \frac{\Delta C}{\Delta y}\right)-\Delta t H_{i, j}^{n}\left(k C_{i, j}^{n}-S_{i, j}^{n}\right)\right\}
$$

in which:

$$
\begin{aligned}
& \Delta(u H C)^{n}=\frac{1-\gamma}{2}\left[(u H C)_{i+1, j}^{n}-(u H C)_{i, j}^{n}\right]-\frac{1+\gamma}{2}\left[(u H C)_{i, j}^{n}-(u H C)_{i-1}^{n}, j\right] \\
& \gamma=1 u>0 ; \gamma=-1 u \leq 0 . \\
& \left(E_{x} H \frac{\Delta C}{\Delta x}\right)^{n}=E_{x i+1, j}^{n} \frac{H_{i, j}^{n}\left(C_{i+1, j}^{n}-C_{i, j}^{n}\right)}{\Delta x}-E_{x i, j}^{n} \frac{H_{i, j}^{n}\left(C_{i, j}^{n}-C_{i-1, j}^{n}\right)}{\Delta x}
\end{aligned}
$$

Boundary conditions: Two different types of external grid interfaces, or boundaries, are possible in the numerical model (5) (7): a water-water or a water-land interface. At the first, the water level $z$ should be specified. At the second, the appropriate normal velocity component is zero.

There are too two types of boundaries in Eq. (8):

$$
\begin{aligned}
& \text { shore boundary: } \frac{\partial C}{\partial n}=0 \\
& \text { water boundary: } \frac{\partial C}{\partial n}+V_{n} \frac{\partial C}{\partial n}=0
\end{aligned}
$$

\section{Application}

\subsection{Simulate the flow of the Pinqing Lake}

The flow of May 2 to May 3,1995,which has the field data, is modeled. At the mouth of Pinqing Lake, the field data of the water level were specified. With the field data of the measured point in Pinqing Lake, the correctness of the model was verified.

The parameters of flow model are $\Delta t=18 \mathrm{~s}, \Delta x=\Delta y=250 \mathrm{~m}, \xi_{x}=\xi_{y}=15 \mathrm{~m}^{2} / \mathrm{s}$, $\eta=0.2$.

The comparison of field data with computed data was in Tab.1. With the table, we can see that the computed data of velocity and flow direction tallied with the field data. So the result which computed from the flow model of Pinqing Lake was feasible. The result of flood was in Fig.2 .The figure shows that after flooding in the lake mouth, the current was divided in two sections: one current is flooding in the north of the channel, the other is flooding in the east of the channel. When ebb (Fig.3), the direction reverses. The current in the lake was smooth and no circulation. 
Tab.1 comparison between calculated and observed values

\begin{tabular}{|c|c|c|c|c|c|c|c|c|c|c|c|c|c|}
\hline Time(h) & 2 & 4 & 6 & 8 & 10 & 12 & 14 & 16 & 18 & 20 & 22 & 24 & 26 \\
\hline pbs u & 39 & 42 & 52 & 62 & 37 & 8 & 39 & 7 & 23 & 11 & 71 & 12 & 40 \\
\hline pbs d & 98 & 254 & 248 & 244 & 93 & 108 & 101 & 54 & 262 & 92 & 88 & 233 & 100 \\
\hline cal u & 33 & 39 & 59 & 57 & 35 & 3 & 36 & 6 & 21 & 48 & 61 & 48 & 25 \\
\hline cal d & 87 & 266 & 265 & 262 & 83 & 82 & 85 & 85 & 266 & 85 & 88 & 267 & 87 \\
\hline
\end{tabular}

$\mathrm{u}$ : flow velocity $\left(\mathrm{cm} \cdot \mathrm{s}^{-1}\right)$; d: flow direction.

\subsection{The prediction of Pinqing Lake water quality}
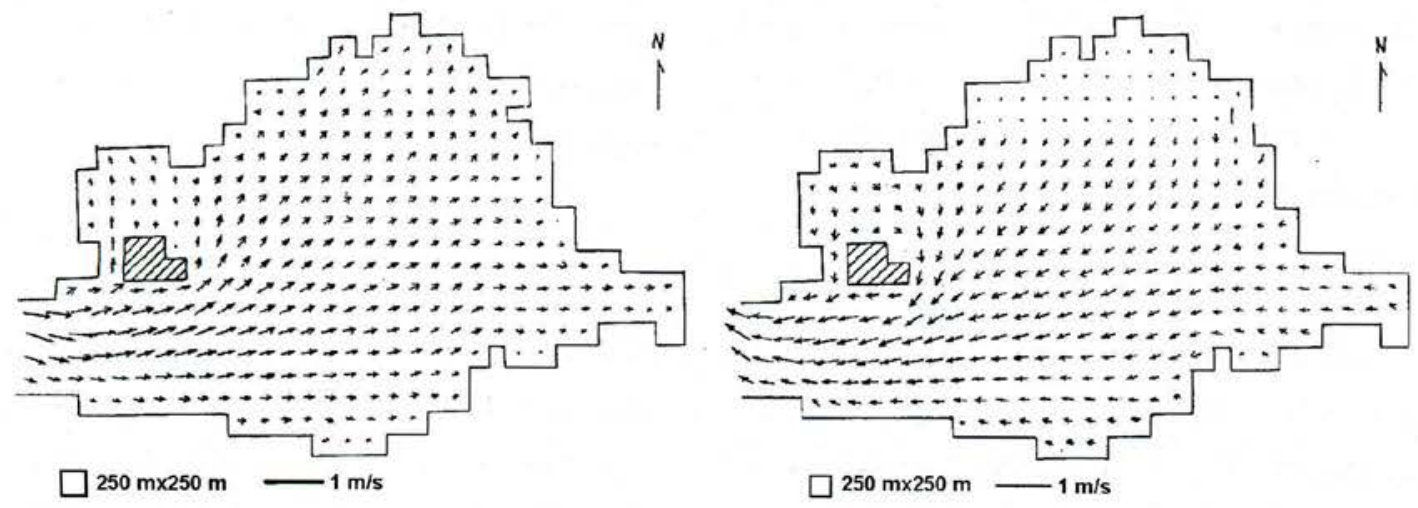

Fig.2 Greatest velocity vectors in flooding

Fig.3 Greatest velocity vectors in ebbing
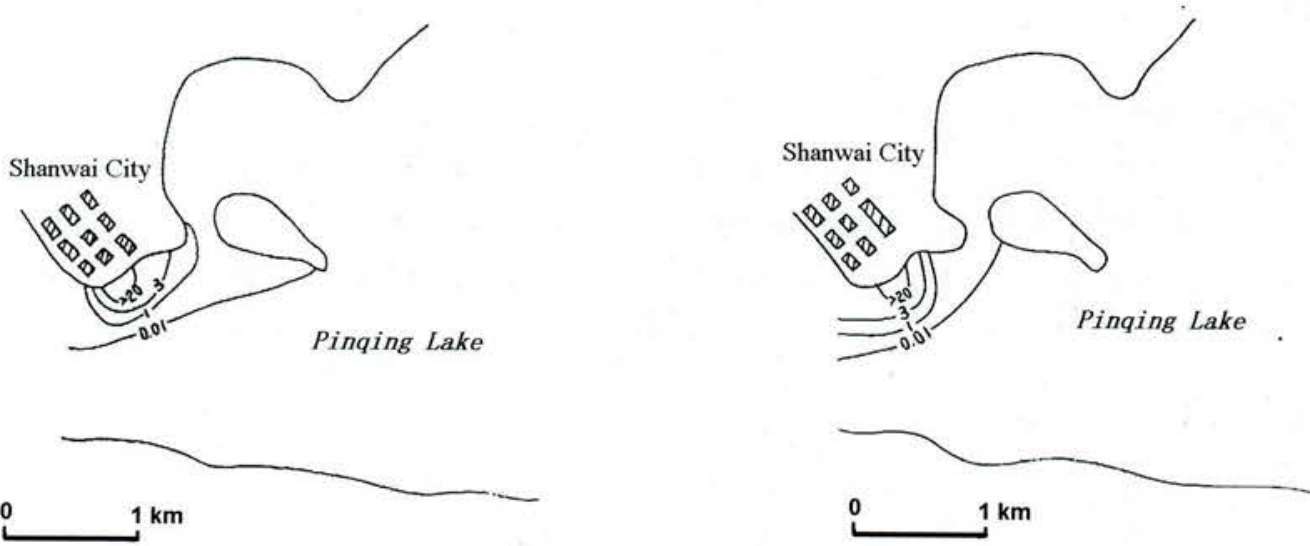

Fig.4 COD concentration contours in flooding.

Fig.5 COD concentration contours in ebbing. 
At present, the concentrations of many pollutants in Pinqing Lake tally with the sea water quality standard I, except the concentration of inorganic nitrogen was higher than the sea water quality standard III and COD concentration of few measured points near the bank were higher than the sea water quality standard II $(C O D>5)$. The mean concentration of COD in the lake is $4.12 \mathrm{mg} \cdot \mathrm{L}^{-1}$.

Equation (8) was used to predict the water quality of Pinqing Lake. It's parameter values as follows. $k=0.08 d^{-1}, E_{x}=E_{x y}=55 \mathrm{~m}^{2} \cdot \mathrm{s}^{-1}, \Delta x=\Delta \mathrm{y}=250 \mathrm{~m}, \Delta t=18 \mathrm{~s}$.

The prediction results were in Fig. 4 and Fig.5.

In discharge points affecting the water quality of Pinqing Lake. Main one is the discharge point of Shanwei City. According to the increase rate of population and the program of industry development, we can count that the discharge capacity of COD from the discharge point of Shanwei City is $253 \mathrm{~g} \cdot \mathrm{s}^{-1}$.

\section{Conclusion}

(1) As the discharge point of Shanwei city is near the mouth of Pinqing Lake, the pollutants go out of the lake when ebbing. The pollutants are obstructed by the small island situated in northeastern part of the discharge point when flooding. They can not go into the center of the lake. So the sewage discharge effect to the water quality of Pinqing Lake is small. But the water quality near the discharge point is bad consequently.

(2) According to the computed data, plus present COD values, the area, where the COD concentration is higher than the sea water quality standard II, is $0.5 \mathrm{sq} . \mathrm{km}$. near the discharge point of Shanwei city. In addition, if we want the area where the COD concentration is higher than the water quality standard II little than 0.2 sq.km., the rate of sewage treatment must be 0.6 . The discharge capacity of COD from the discharge point of Shanwei city should be reduced to $152 \mathrm{~g} \cdot \mathrm{s}^{-1}$.

\section{References}

Huang, P. 1996. Three-dimensional numerical simulation of the heat diffusion from the cooling water in Shantou harbour. Marine Environmental Science.1:59-65.

Huang, P., Mao, R. S. 1997. A 3-dimension implicit finite-difference model for wind-driven water current in Moshui Lake. J. Lake Sciences. 9: 15-21. 